\title{
A pre-reflective indicator of an impaired sense of agency in patients with Schizophrenia
}

\author{
Virginie Bulot $\cdot$ Pierre Thomas \\ Yvonne Delevoye-Turrell
}

Received: 12 October 2006 / Accepted: 12 June 2007 / Published online: 10 July 2007

(C) Springer-Verlag 2007

\begin{abstract}
In schizophrenia, passivity phenomenon are clinically related to an abnormal sense of agency, which has been experimentally studied through self-recognition tasks. However, Tsakiris et al. (Cognition 96(3):215-231, 2005) have recently shown in healthy controls that the sense of agency is distinct from self-recognition abilities. We propose a simple motor task to obtain an implicit indicator of the working status of the pre-reflective sense of agency in schizophrenia. Collision dynamics gave us the means to further dissociate agency from motor prediction. Twenty-four patients and a group of matched controls used a hand-held object to stop the fall of a pendulum that was released either by the Subject (task S) or by the Experimenter (task E). The objective indicator of the sense of agency was taken as the efficiency difference between tasks $\mathrm{S}$ and $\mathrm{E}$, before the availability of afferent information from collision. Qualitative feedback was provided to assess the top-down effect of explicit information. Motor prediction was as accurate in patients as in controls in tasks $\mathrm{E}$ and $\mathrm{S}$.
\end{abstract}

Study conducted in the Psychiatric Unit - University Regional Hospitals of Lille (France).

V. Bulot

Service de Psychiatrie Adulte Unité 72,

Hôpital André Mignot, 177 route de Versailles,

78150 Le Chesnay, France

P. Thomas

CNRS UMR8160, Université Lille 2,

CHRU Lille, 6 rue Pr. Laguesse, 59037 Lille Cedex, France

Y. Delevoye-Turrell ( $\square)$

Laboratoire URECA-UFR de Psychologie,

Université de Lille3, rue du Barreau,

59653 Villeneuve d'Ascq Cedex, France

e-mail: yvonne.delevoye@free.fr
Controls were more efficient in $\mathrm{S}$ than in E. Patients revealed similar efficiency levels in both tasks. Qualitative feedback helped but did not affect the efficiency difference between tasks. Our results suggest an impairment of a pure efferent-driven sense of agency in schizophrenia, which is (1) distinct from motor prediction and (2) not under voluntary control. The abnormal judgments previously reported in schizophrenia for self-recognition abilities might be the consequence of a low order deficit of a pre-reflective sense of agency.

Keywords Schizophrenia - Agency · Motor awareness · Efficiency · Prediction · Judgments of attribution .

Feedback · Collision · Grip force

\section{Introduction}

The sense of agency is a complex phenomenon that draws upon a variety of agency indicators (Wegner and Sparrow 2004). The topic of the current research is the sub conscious sense of agency that has been described as the immediate experience of oneself as the cause of an action (Gallargher 2000). Because of its pre-reflective nature, it has been considered as a formal part of the minimal self (Gallargher 2004). In pathological cases, it has been suggested that auditory hallucinations, thought insertions and delusion of influence, i.e. passivity phenomena, may be caused by a distorted sense of agency (Frith 1992). Frith and colleagues conducted several experimental studies and provided results that suggested that patients with schizophrenia misattribute self-initiated actions as externally initiated actions. It is true that these errors in judgments of attribution may be caused by an abnormal sense of agency and the possibility that the "immunity to error principle" 
(Shoemaker 1984) for the minimal self may not be verified in schizophrenia motivated multiple experimental studies testing the functional status of the sense of agency in these patients. However, the experimental methods used to date do not provide the means to dissociate a distorted sense of agency from problems in judgments of attribution. The aim of the work presented here was to test patients with schizophrenia with an objective and implicit behavioural task in order to test directly the working status of the sense of agency in schizophrenia.

The challenging theoretical proposition made by Frith and co-workers (e.g. Frith et al. 2000) was that an abnormal sense of agency could be the direct cause of the principle symptoms characterizing schizophrenia, e.g. delusions of control and auditory hallucinations. Indeed, patients would not experience themselves as the author of their own actions. To explore this specific problem, a series of experimental studies were run using various self-recognition tasks. For instance, Daprati et al. (1997) asked subjects to judge whether a movement shown on a computer screen was one they had previously performed or not. Results showed that patients with passivity phenomena had false judgements of attribution $80 \%$ of the time under the condition where the visual feedback was very finely distorted, whereas healthy controls were induced to error only $30 \%$ of the time. This study and others (Franck et al. 2001) suggest that patients suffering from passivity phenomena have difficulties in performing self-recognition tasks. This may be a core deficit in schizophrenia and a better understanding of the impairment could provide important insights in the psychopathology of schizophrenia. However, because (1) the reflective nature of these judgment tasks only was considered and because (2) the subjects were under all conditions the authors of the actions (i.e. only self-generated movements were employed), only the explicit level of self-recognition was explored; in no case was considered the level of "immediate experience" for the sense of agency.

Frith and colleagues attempted to explore the sense of agency more specifically and proposed a cognitive model based on motor prediction (Frith 1992; Frith et al. 2000; Blakemore et al. 2002). At the core of their model lies the concept of "efference copy" (Sperry 1950; Von Holst and Mittelstaedt 1950, cited in Nelson 1996; Feinberg 1978). Here, a copy of the motor command would provide the motor system with the means to predict and anticipate the sensory consequences of self-initiated actions. Awareness of own actions would largely be dependent on the outcome of the comparison between the predicted and the actual states of movements, and this comparison would provide the signal necessary for sensory attenuation (Blakemore et al. 2002). Supporting this theory, healthy subjects were shown to judge self-initiated events as less intense than externally initiated ones (Shergill et al. 2003 for force pulses). In a ticklish study, it was shown that healthy controls judged self-initiated tactile stimulations as being less ticklish than externally initiated ones (Blakemore et al. 1998), whereas patients with passivity phenomenon judged self-initiated and externally initiated tactile stimulations as being as ticklish (Blakemore et al. 2000). Thus, it was proposed that the absence of sensory attenuation for self-initiated actions in schizophrenia would be due to impaired forward model predictions. More specifically, patients would not be able to distinguish self-initiated from externally initiated actions and thus, they would experience passivity phenomenon. Similar conclusions have been recently presented in a force-matching task (Shergill et al. 2005). To note is the fact that the sense of agency was explored here at the immediate level of experience. Nevertheless, it was once more assessed through the means of a reflective selfreport, and because of the pre-defined theoretical framework, agency was considered as being dependent only on forward model motor predictions.

Tsakiris and Haggard (2003) have recently shown that healthy controls possess an attenuated sensory feedback of self-initiated actions even when motor prediction about the intensity of the self-initiated effect is not possible. Thus, if the sense of agency arises with sensory attenuation then, it cannot be directly and only be related to forward model predictions. To test this hypothesis, they performed a study in which participants experienced a passive extension of the right index finger, either as an effect of moving their left hand via a level, or imposed externally by the experimenter. Visual feedback was manipulated so that participants saw the passive extension of either their own right index finger or someone else's right index finger. The participants' task was to judge whether the right finger they saw was theirs or not. Self-recognition performance was more accurate in the self-generated action condition, and dropped to near chance levels when only proprioceptive afferent information was available. Thus, efferent information from self-generated actions might provide a precise temporal signal, which could be used to better predict proprioceptive and/or visual information. From these results, Tsakiris and Haggard (2005) developed the concept of an efferent-driven sense of agency and proposed that sensory attenuation may be a "sensory bias" and that "The mere presence of intention and 'raw' efferent information suffice for this sensory bias to be generated". Accordingly, there would be two types of sensory attenuation that would play a critical role in distinguishing motor prediction from the sense of agency. First, there would be a proportional sensory attenuation that would be a direct result of motor prediction; the sensory attenuation would be in function of the degree of discrepancy between the predicted and the true afferent information from action generation (post-action). Second, there would be a fixed sensory attenuation that would result from 
efferent information only, which would function as a general contextual modulation (pre-action). This input contextual parameter could be integrated within the process of motor planning and as such, should be experimentally observable very early during action planning.

The key question arising from these previously mentioned studies is the possibility that pre-reflective agency, a sense that would be based on efferent information only, would have a bottom-up effect on self-recognition tasks. In such a case, could the deficits in self-recognition tasks previously reported in schizophrenia not be a direct consequence of low order dysfunctional mechanisms of a pre-reflective sense of agency? Even if recent data have provided promising results in this direction in healthy controls (Tsakiris et al. 2005), it has not yet been explored in patents with schizophrenia.

In the present study, our aim was to explore specifically the possibility of the existence of an efferent-driven sense of agency in healthy controls and to assess whether this sense of agency is impaired in patients with schizophrenia when required to experience self-initiated versus externally initiated actions in the event of a collision. The key point was the fact that we used a simple motor task that required no reflective response or judgment and consequently, we explored the sense of agency at the immediate level of experience, through the means of an implicit task. In addition, because of the impulsive nature of collisions (Turrell et al. 2001), our approach provided the means to examine in schizophrenia the problem of agency and of motor prediction in a pure efferent-driven situation. Our results will show that the use of efferences for forward model predictions as well as the integration of afferent and efferent information for motor learning is normal in schizophrenia; the only abnormal finding is the patients' pre-reflective experience of being the agent of self-initiated collisions.

\section{Methods}

Subjects

Twenty-four right-handed inpatients (full time or day hospitalisation), compelling to the DSM IV criteria for paranoid schizophrenia and 24 right-handed healthy volunteers, pair matched for sex and age, participated in the study. Patients underwent a detailed clinical assessment (see Table 1 for demographic and clinical data). Exclusion criteria for all subjects were: history of head trauma, neurological disease or substance abuse/dependence (DSM IV), presence of fine sensori-motor integration deficits. The controls were recruited through local advertisement and were further screened for history of psychotic disease and/or episode, and of possessing a first-degree relative with such sdifficulties. For this study, 29 schizophrenic inpatients were contacted, 2 refused and 3 were excluded before performing the experimental tasks. 32 controls were contacted, 4 refused and 4 were excluded. The protocol was approved by the local ethic committee and after complete description of the procedure to the subjects, written informed consent was obtained.

\section{Apparatus}

Subjects faced a table upon which was mounted a metal frame (height $2 \mathrm{~m}$; width $1 \mathrm{~m}$; depth $80 \mathrm{~cm}$ ). This frame was the support for a pendulum (length $75 \mathrm{~cm}$; weight $750 \mathrm{~g}$ ) made of an aluminium rod and of a hard-rubber head; the pendulum swung along the subject's frontal-axis only. Subjects held in their right hand a 6-axis load cell (Novatech Gamma SI-130-10) with parallel sandpapercovered surfaces (width $4.5 \mathrm{~cm}$; weight $450 \mathrm{~g}$ ), which measured the tangential and the normal forces in Newton (N) applied to its surfaces throughout each trial (Fig. 1a, b). A potentiometer (10-K turn wire wound RS173-417) at the centre of rotation of the pendulum measured the angle of pendulum position $\left(0^{\circ}\right.$ being the vertical position $)$, information that was used to set the angle of pendulum release at the start of each trial.

\section{Peripheral sensorimotor screening}

All subjects were screened for peripheral motor deficits by measuring the timing and amplitude of the long-latency reflexes. Subjects were required to grip the load cell with a precision grip, with their right hand at the pendulum resting position and with their eyes closed. Subjects were told that the pendulum would be released and they were instructed to stop the pendulum's fall without moving their hand back or letting the load cell slip within grip. The subjects could employ whatever grip force level they wanted to. They were not aware of the moment or of the degree of pendulum release. Reflex time delays were similar $(P>0.05)$ in controls (98 SD $11 \mathrm{~ms}$ ) and in patients with schizophrenia (91 SD $12 \mathrm{~ms}$ ).

\section{Experimental procedure}

After washing and drying their hands, subjects were asked to stand in front of the pendulum, in a stable upright posture. They were required to arrest the fall of the pendulum by using a precision grip to hold the load cell (object) immobile at the pendulum's resting position $\left(0^{\circ}\right)$. Subjects were instructed to be the most efficient possible i.e. use the smallest amount of grip force at the time of impact, without letting the object slip within the fingers. Subjects had their eyes open and performed two different tasks of 36 blocked trials each (12 consecutive trials per randomised angle). 
Table 1 Presents the demographic and clinical data (means) for the patients and the controls who received $(\mathrm{F}+)$ or not $(\mathrm{F}-)$ qualitative feedback about efficiency performance after each trial

\begin{tabular}{|c|c|c|c|c|c|c|c|c|}
\hline \multirow[t]{2}{*}{ Within group comparison } & \multicolumn{4}{|c|}{ Patients } & \multicolumn{4}{|c|}{ Controls } \\
\hline & $\mathrm{F}-$ & $\mathrm{F}+$ & $t$ & $P$ & $\mathrm{~F}-$ & $\mathrm{F}+$ & $t$ & $P$ \\
\hline Age & 36.7 & 36.6 & -0.024 & 0.983 & 35.7 & 35.6 & 0.016 & 0.982 \\
\hline Sex & 1.2 & 1.4 & -0.726 & 0.482 & 1.3 & 1.3 & 0.432 & 0.671 \\
\hline Stroop (Interference score) & -2.4 & -4.9 & 0.669 & 0.509 & 4.5 & 2.6 & 0.684 & 0.501 \\
\hline TMT A & 47.4 & 47 & 0.062 & 0.951 & 27.4 & 23.3 & 1.223 & 0.244 \\
\hline TMT B & 139.8 & 141.9 & -0.053 & 0.950 & 56.6 & 52.4 & 0.569 & 0.601 \\
\hline WCST serial & 3.6 & 3.11 & 0.678 & 0.499 & 5.4 & 5.6 & -0.443 & 0.662 \\
\hline WCST \% correct & 61.2 & 59.7 & 0.162 & 0.873 & 58.7 & 72.4 & -1.986 & 0.289 \\
\hline WCST \% rules break & 4.7 & 5.3 & 0.318 & 0.754 & 1.9 & 1.2 & 0.708 & 0.483 \\
\hline WCST \% perseveration & 10.6 & 11.1 & 0.087 & 0.93 & 2.1 & 2.3 & -0.149 & 0.882 \\
\hline WCST \% error & 24.7 & 23.8 & 0.181 & 0.859 & 7.6 & 8.4 & -0.583 & 0.560 \\
\hline Illness duration & 13.5 & 15.4 & -0.493 & 0.624 & & & & \\
\hline Haloperidol/day (mg) & 9.4 & 8.8 & 0.286 & 0.782 & & & & \\
\hline Prazépam/day (mg) & 41.8 & 29 & 1.221 & 0.236 & & & & \\
\hline PANSS + & 24 & 20 & 1.711 & 0.113 & & & & \\
\hline PANSS - & 16.8 & 19.5 & -1.063 & 0.300 & & & & \\
\hline PANSS general & 36.3 & 35.4 & 0.291 & 0.771 & & & & \\
\hline
\end{tabular}

The group mean scores for the different clinical tests that were run are also presented. The last two columns present the statistics for the $t$ test and the significance level $P$ that was conducted and which failed to reveal any significant within group effects. The Wisconsin Card Sorting Test (WCST) is a test evaluating the quality of executive function; the Stroop test is a test evaluating a specific attentional deficit in inhibitory capacities; the Trail Making Test (TMT A and B) is a test assessing selective attention; the Positive and Negative Syndrome Scale (PANSS) is a clinical scale evaluating the intensity of positive and negative schizophrenic symptoms; the Simpson and Angus Scale evaluates the intensity of extrapyramidal symptoms; the Buchanan and Heinrich Scale reveals neurological soft signs in schizophrenia, with a total score (0-88) and 3 partial scores : sensorial integration (0-14), motor coordination (0-14), dynamic praxia (=complex motor sequences : $0-12)$. Treatment posology are converted in haloperidol unit equivalency or in prazepam unit equivalency per day

Task E

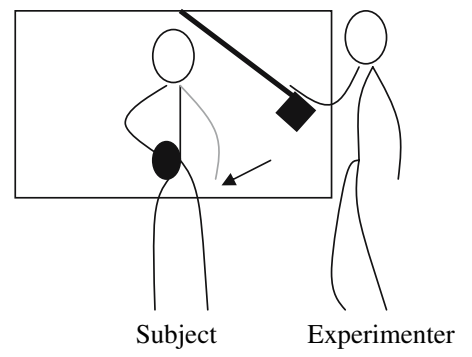

Task S

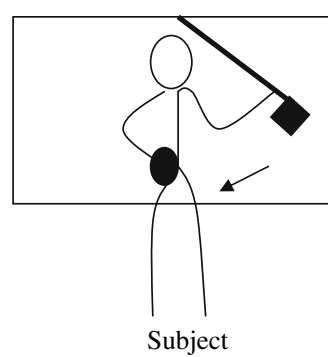

Fig. 1 Is an illustration of a subject holding a load cell (dark circle) in his/her right dominant hand at the pendulum's resting position. The subject's task was to stop the fall of the pendulum while being the most efficient possible at the critical time of impact, i.e. apply the smallest amount of excess grip force on the load cell, without letting it slip within grasp. In task E (left), the pendulum was released by the experimenter. In task S (right), the pendulum was released by the subject. With this setup, the only difference between tasks $\mathrm{E}$ and $\mathrm{S}$ was the agent of pendulum release

- Task E (the Experimenter was the agent, Fig. 1 left): The experimenter placed the pendulum at the pre-set starting angle of $15^{\circ}, 25^{\circ}$ or $35^{\circ}$. The subjects' task was to arrest the pendulum fall with the object held in their right hand.
The experimenter released the pendulum at the sound of an auditory stimulus.

- Task S (the Subject was the agent, Fig. 1 right): Subjects used their left hand to place the pendulum at the pre-set starting angle of $15^{\circ}, 25^{\circ}$ or $35^{\circ}$. Their task was to arrest the pendulum fall with the object held in their right hand. Subjects were required to release the pendulum at the sound of an auditory stimulus.

Because of the nerve conduction delays, afferences are available only after a time interval of at least $30 \mathrm{~ms}$. With the present setup, the impact between the hand-held load cell (object) and the pendulum lasted between 10 and $15 \mathrm{~ms}$, in both tasks E and S (Turrell 2000). Thus, to succeed in these tasks, the grip force applied on the object had to be increased before collision in order to prevent objectslip at the critical time of impact (Delevoye-Turrell et al. 2001). Since grip force level was set by the subject prior to the availability of afferent information from impact, the grip force measured at the very time of impact was considered to be purely predictive in nature.

Patients and controls were randomly assigned to two different experimental subgroups. In the feedback subgroup 
$(\mathbf{F}+)$, subjects received explicit feedback of the efficiency level measured at the time of impact. This feedback was given after each trial by means of a visual vertical analogical scale $(10 \mathrm{~cm}$ long; top: "way too much excess grip force"-bottom: perfect, no excess grip force). Subjects in the no-feedback subgroup $(\mathbf{F}-)$ performed both tasks without supplemented information about performance efficiency. Experimental subgroups were statistically comparable for age, sex and scores, for the selected cognitive tests (see Table 1). After each trial and for all groups, subjects were asked to indicate on the visual vertical analogical scale the level of efficiency they thought they had reached.

\section{Data analyses}

For each of the 72 experimental trials, grip force (GF) and load force at impact (LF) were automatically scored using Labview programs. The following computations were performed.

\section{Accuracy of anticipatory adjustments}

Grip force was plotted against LF for each subject and task. The effects of Group and Task on the regression coefficients (correlation, slope and intercept) were then examined. Results revealed that the intercept statistics were identical to those observed for the efficiency of the anticipatory adjustments. Consequently, intercept results are not reported.

\section{Efficiency of anticipatory adjustments}

In the literature, the relative safety margin has been used as a quantitative measure of the efficiency of GF to LF scaling (Westling and Johansson 1984; Delevoye-Turrell et al. 2003a).

The calculation of the safety margin requires the estimation of the minimum GF to LF ratio needed to prevent slip, i.e. the slip ratio. In the present study, the slip ratio was estimated for each subject at the start of the session by asking subjects to voluntarily hold the load cell with the lowest GF possible. Then, the individuals' slip ratio were taken as the ratio measured between GF and LF on those trials during which the load cell slipped within the subjects' grip as a consequence of the impact. Ten trials per subject were recorded and the mean individual slip ratios were calculated. Values ranged from 0.04 to 0.18 (see DelevoyeTurrell et al. 2001 for comparative values).

The relative safety margin (SM) was then calculated and used as an indicator of the excess GF employed at the time of impact. It was defined as the fractional amount by which the GF to LF ratio exceeded the critical individual slip ratio. SM was determined for each subject and on each trial, according to the equation: Safety Margin $\mathrm{SM}=[\mathrm{GF} / \mathrm{LF}] /$ slip ratio $\times 100 \%$. For instance, a SM of $100 \%$ would be an optimal result as it suggests that subjects used a GF level equal to the minimal GF necessary to avoid object slip. A SM of 500\% would however indicate poor performance as it suggests that subjects used 5 times more GF than truly necessary.

\section{Statistical analyses}

To ensure equivalency between the levels of performance in patients with schizophrenia and controls, (1) mean impact force and mean GF at impact were run through a 2 (Group: patients; controls) $\times 3$ (Angle: $\left.15^{\circ} ; 25^{\circ} ; 35^{\circ}\right) \times 2$ (Task: E;S) repeated measures ANOVA. Mean time delay between impact and GF peak was run through a 2 (Group) $\times 2$ (Task) repeated measures ANOVA.

The accuracy of motor prediction was assessed by running the means of the regression coefficients through a 2 (Group) $\times 2$ (Feedback: F+; F-) $\times 2$ (Task) repeated measures ANOVA. The efficiency of motor prediction was assessed by running the SM means through a 2 (Group) $\times 2$ $($ Feedback $) \times 2$ (Task) repeated measures ANOVA.

To reveal a possible effect of learning, a fourth analysis was conducted to assess the changes in GF efficiency between the first 3 trials and the last 3 trials of each series of 12 trials (Time factor). When required, Fisher post hoc analyses were run with Bonferroni corrections for multiple comparisons.

Finally, separate correlation values were computed for each task and each experimental group between the subjective and objective GF efficiency levels reached at the time of impact, in order to examine possible differences of awareness in the levels of performance reached by the controls and by the patients with schizophrenia.

In a final section, we present a subdivision of the patient group that we performed for each of the clinical scores around the median score, in order to assess whether the patients' results could be considered as homogenous. The independent variable that was used was the range of criterion score (low or high). A Student's $t$ test was then run to assess a possible sub-group discrepancy on the efficiency difference between tasks $\mathrm{E}$ and $\mathrm{S}$ (taken as the dependant variable). Correlation tests were also run between the clinical scores and the efficiency difference between tasks $\mathrm{E}$ and $\mathrm{S}$.

\section{Results}

Patients and controls performed the experimental tasks without apparent difficulty. Subjects were frequently asked who was releasing the pendulum, and none of the participants 
made errors of agent attribution at this reflective and explicit level.

\section{Analysis 1: Equivalency of behaviour}

Impact forces were significantly different for the three angles of $15^{\circ}, 25^{\circ}$ and $35^{\circ}, F(2,45)=4248.6, P=0.000$. They were similar for controls and patients, $F(1,45)=0.102, P=0.890$ : 13.1 N, 24.7 N, 35.5 N and 13.6 N, 24.6 N, 35.1 N for controls and patients, respectively. These impact forces afforded GF levels at impact of $4.6 \mathrm{~N}, 6.8 \mathrm{~N}, 10.3 \mathrm{~N}$ and $6.1 \mathrm{~N}, 8.2 \mathrm{~N}$, $11.3 \mathrm{~N}$ for controls and patients, respectively. Statistically, mean GF at impact was significantly different for the three impact forces, $F(2,45)=189.210, P=0.000$. The interaction Group $\times$ Angle was not significant, $F(1,45)=2.844$, $P=0.092$.

The time delay between impact and peak GF was similar for controls (58 SD $4 \mathrm{~ms}$ ) and patients (69 SD $5 \mathrm{~ms}$ ), $F(1,45)=1.386, P=0.239$. It was significantly shorter in task S (44 SD $3 \mathrm{~ms}$ ) than in task $\mathrm{E}(73 \mathrm{SD} 5 \mathrm{~ms})$, $F(1,45)=8.550, P=0.003$. The interaction Group $\times$ Task was not significant, $F(1,45)=0.132, P=0.716$. No other effects were significant.

Overall, these results indicate that (1) both experimental groups experienced collisions of similar magnitudes and (2) that patients and controls were able to perform tasks $\mathrm{E}$ and $\mathrm{S}$ following instruction by scaling grip force in anticipation of forthcoming collisions. The timing of the anticipatory GF increase with impact was as accurate in patients as in controls, and both groups took advantage of self-initiating the pendulum release (with the left hand) in order to improve the timing of the GF increase (of the right hand).

Analysis 2: Accuracy of motor prediction

ANOVAs on the correlation of the individual subjects' regression lines were run. GF was significantly correlated with LF, and at a similar degree, in controls (task E: 0.501, range $=0.417-0.585$; task $\mathrm{S}: 0.613$, range $=0.548-0.677$ ) and in patients with schizophrenia (task E: 0.523, range $=0.428-0.618$; task $S: 0.604$, range $=0.515-0.694)$, $F(1,42)=1.255, P=0.268$. The correlation values were statistically similar in task S (0.607 SD 0.184) and in task E $(0.512$ SD 0.208), $F(1,42)=1.109, P=0.298$. The interaction Group $\times$ Task was not significant, $F(1,42)=0.804$, $P=0.453$.

ANOVAs on the slope (extent of GF modulation) of the individual subjects' regression lines were run. The slopes of the regression lines were different from 0 for all subjects, and were similar in controls (task E: 0.192, range $=0.128-$ 0.256 ; task $S: 0.336$, range $=0.158-0.514$ ) and in patients (task E: 0.225 , range $=0.106-0.344$; task $\mathrm{S}$ : 0.338 , range $=0.178-0.497), F(1,42)=0.231, P=0.633$. Slopes were similar in task $\mathrm{S}(0.332 \mathrm{SD} 0.214)$ and in task $\mathrm{E}$ $(0.208$ SD 0.318), $F(1,42)=2.174, P=0.147$. The interaction Group $\times$ Task was not significant, $F(1,42)=0.723$, $P=0.550$.

These results indicate an absence of task effect on the accuracy of the predicted GF adjustments for both patients and controls. It suggests that motor prediction is as accurate in task $\mathrm{S}$ as in task $\mathrm{E}$ for both experimental groups.

Analysis 3: Efficiency of motor prediction

The following results are presented in Fig. 2. ANOVA on SM means revealed that patients were overall less efficient (447 SD 273\%) than controls (405 SD 202\%), $F(1,45)=$ $6.116, P=0.014$. The Group $\times$ Task interaction was significant, $F(1,45)=5.636, P=0.018$. Indeed, for the controls, the efficiency difference between tasks $\mathrm{E}$ and $\mathrm{S}$ was significant: GF efficiency was greater in task S (380 SD 169\%) than in task E (431 SD 235\%), $F(1,45)=6.116, P=0.003$, affording an efficiency difference of $51 \%$. For the patients, there was no significant efficiency difference between the two tasks: GF efficiency was similar in task S (449 SD 230\%) and in task E (442 SD 324\%), $F(1,45)=6.116, P=0.669$, affording an efficiency difference of $-7 \%$. GF efficiency in task $\mathrm{E}$ was statistically similar for controls and patients, $F(1,45)=$ 6.116, $P=0.653$.
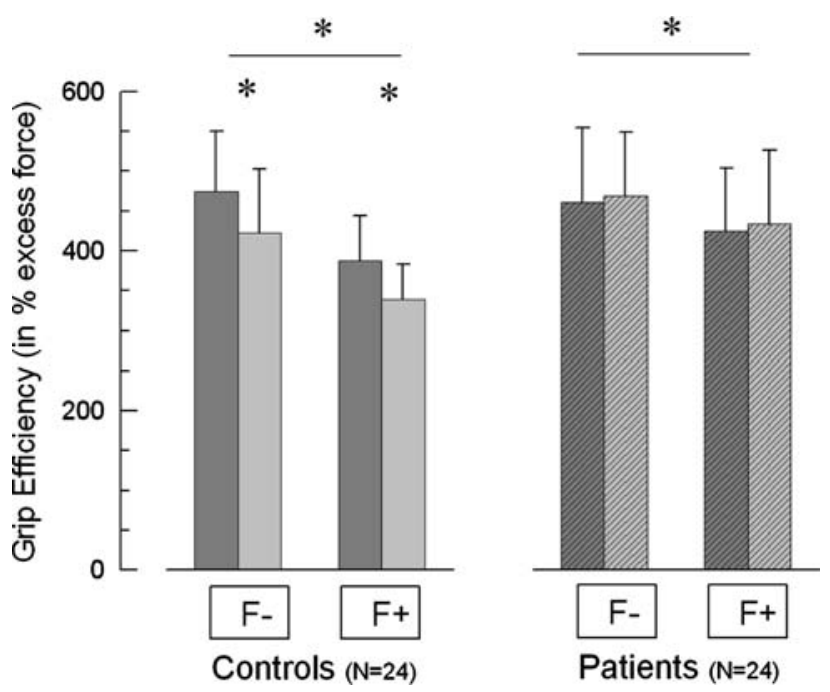

Fig. 2 Illustrates the Task $\times$ Group interaction for the percentage of excess GF used at the time of impact. Mean and standard deviation (bars) are presented for the control subjects (left) and the patients with schizophrenia (right) performing task $\mathrm{S}$ (light grey) and task E (dark grey), with $(\mathrm{F}+)$ or without $(\mathrm{F}-)$ qualitative feedback about performance efficiency. One can note that qualitative feedback improves the efficiency level for all groups. However, under both feedback conditions, control subjects are more efficient in task $\mathrm{S}$ than in task $\mathrm{E}$, whereas patients with schizophrenia reveal similar levels of efficiency in both tasks. The efficiency levels differ between experimental groups only for task $\mathrm{S}$, for which patients are significantly and consistently less efficient than matched controls 
When considering the main effect of Feedback, results revealed that subjects were overall more efficient with feedback (396 SD 174\%) than without (456 SD 285\%), $F(1,45)$ $=13.038, P<0.001$. Neither the Group $\times$ Feedback interaction, $F(1,45)=2.250, P=0.134$, nor the Group $\times$ Feedback $\times$ Task interaction, $F(1,45)=0.002, P=0.968$, were significant.

Analysis 4: Learning effects and stability of the efficiency difference between tasks $\mathrm{E}$ and $\mathrm{S}$

Figure 3 illustrates the learning effects that took place for controls (top) and patients with schizophrenia (bottom) in task E (triangle) and in task S (circles). When comparing the first three trials with the last three trials of each series, results revealed a significant main effect of Time, $F(1,45)=76.75, P<0.001$ : GF efficiency was better at the end (378 SD 192\%) than at the start (526 SD 336\%). The Group $\times$ Time interaction was not significant, $F(1,45)=$ $0.868, P=0.352$, revealing that both control subjects (493 vs. $361 \%$ ) and patients (562 vs. $397 \%$ ) improved through time their efficiency levels, at a similar degree. Finally, the Group $\times$ Task $\times$ Time interaction was not significant, $F(1,45)=0.050, \quad P=0.815$, indicating that efficiency difference between tasks $\mathrm{E}$ and $\mathrm{S}$ was similar at the start and at the end of the learning phase, and that this was true in both experimental groups.

Analysis 5: Correlation values indicator of the subjects' awareness of motor efficiency

Results showed that all subjects possess an awareness of performance above chance level. For the control group, the correlation values between the objective GF efficiency measure and the subjects' subjective appreciation of efficiency was greater in task S (0.40) than in task E (0.24). This suggested that healthy controls possessed a greater awareness of their motor performance in task S. For the patients group, the correlation values were similar in task $\mathrm{S}$ $(0.24)$ and in task E (0.22), suggesting that patients with schizophrenia possess similar levels of awareness for GF efficiency whether they are agent of pendulum release or not. Once more, results were similar in task E in controls and in patients with schizophrenia.

Analysis 6: Homogeneity within the patient group

Results revealed that none of the clinical criteria caused heterogeneity within the patients' results: whether with high or low clinical scores, patients revealed a similar absence of efficiency difference between tasks $\mathrm{E}$ and $\mathrm{S}$ (columns 1 and 2 of Table 2). In addition, no significant correlations between the clinical scores and the efficiency
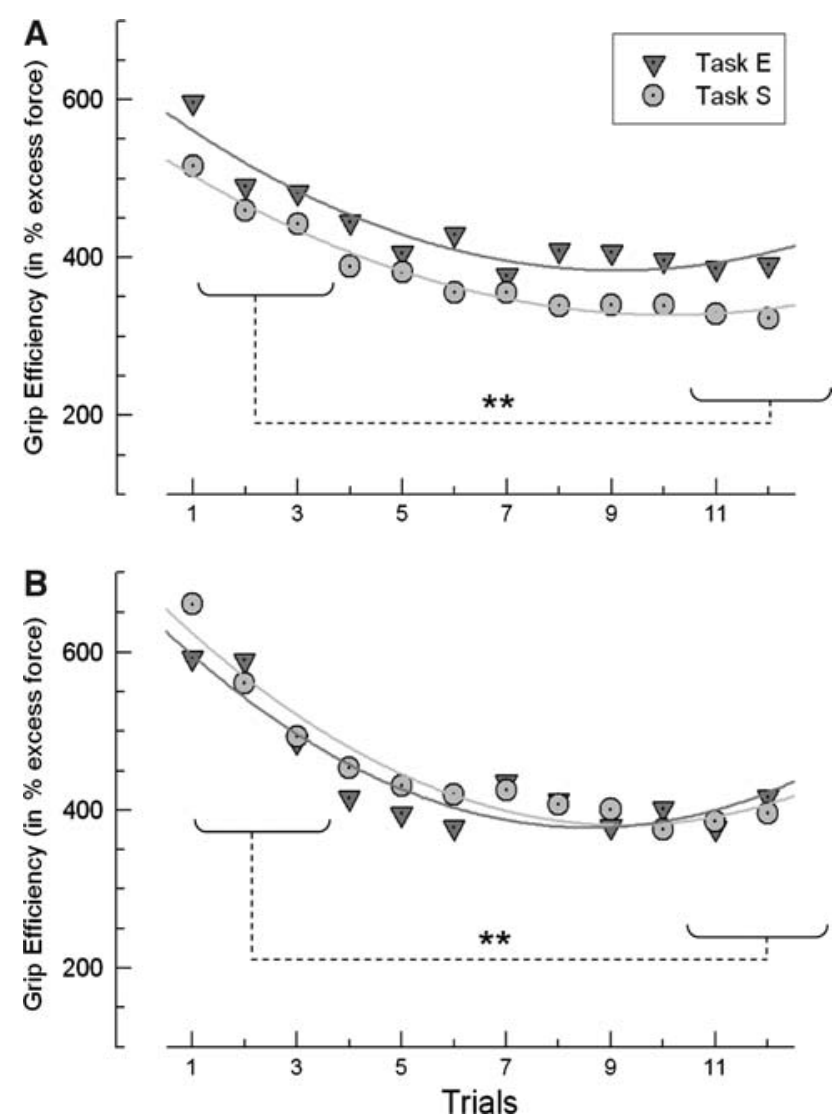

Fig. 3 Reports the time series that reveal the improvement of GF efficiency for (a) control subjects and (b) patients with schizophrenia, when performing task $\mathrm{E}$ (dark grey triangles) and task S (light grey circles). As revealed by the slopes of the learning curves, learning rates were similar both across tasks and across groups. Hence, the absence of difference between tasks $\mathrm{E}$ and $\mathrm{S}$ in patients with schizophrenia cannot be associated to a problem in motor learning

difference between tasks $\mathrm{E}$ and $\mathrm{S}$ were revealed (column 3 of Table 2).

\section{Discussion}

In the last decade, most studies intending to study the sense of agency may have used experimental methods that rather pin pointed judgments of attribution. Indeed, these topdown procedures required participants to judge explicitly whether they were, or not, author of an action. Our aim here was to develop an objective and simple behavioural probe that would provide the means to examine the pre-reflective sense of agency, i.e. the immediate experience of oneself as the initiator of an event (Gallagher 2004). Specifically, we designed a task that required neither reflection nor judgment. In the present study, the subjects' task was to be as efficient as possible while arresting the fall of a pendulum that was either initiated by the experimenter (Task E) or by the subject (Task S). The efficiency level used by the 
Table 2 presents the statistical comparison between the efficiency difference between task $\mathbf{E}$ and $\mathbf{S}$, for those patients who presented low scores $(N=12)$ and high scores $(N=12)$ in the different clinical scores presented in Table 1

\begin{tabular}{lrrc}
\hline \multicolumn{1}{c}{$t$} & $P$ & $R$ \\
\hline $\begin{array}{l}\text { Results of homogeneity for patients with schizophrenia } \\
\quad(N=24,12 \text { per group })\end{array}$ & & \\
Age & 1.012 & 0.322 & -0.19 \\
Illness duration & 1.012 & 0.322 & -0.19 \\
PANSS + & -1.112 & 0.281 & 0.15 \\
PANSS - & -1.160 & 0.264 & -0.2 \\
Simpson and Angus scale & -1.013 & 0.320 & -0.17 \\
Buchanan Sensory integration & 1.290 & 0.232 & 0.32 \\
Buchanan Motor coordination & -1.000 & 0.320 & -0.15 \\
Buchanan Dynamic praxia & 0.789 & 0.443 & 0.20 \\
Dose of haloperidol/day & 0.226 & 0.822 & 0.18 \\
Dose of parazepam/day & 0.396 & 0.693 & -0.005 \\
Delusion of control & 0.928 & 0.366 & -0.27 \\
Cognitive scores ( $z$ score for all tests $)$ & 1.551 & 0.131 & -0.23 \\
\hline
\end{tabular}

Results revealed an absence of group difference (columns 1 and 2), which suggests that our results are not attributed to heterogeneity problems within the patients group. Furthermore, the Pearson correlation values (column 3) revealed no significant correlations between the clinical scores and the efficiency difference between tasks $\mathrm{E}$ and $\mathrm{S}$

subject at the critical time of collision was taken as the indicator of his/her immediate experience of that collision. Results revealed that healthy controls were significantly more efficient when the pendulum release was self-initiated (task S) than when it was released by the experimenter (task E), suggesting that healthy individuals pre-reflectively experience self-initiated events as different from externally initiated ones. Patients with paranoid schizophrenia on the other hand set similar efficiency levels whoever the agent of the release was. This finding suggests that, at the level of immediate experience, patients experience similarly selfinitiated and externally initiated actions.

An essential aspect to remember about our study is the fact that the collision paradigm provides the means to dissociate two parameters that are confounded in most studies: (1) motor prediction based on efference copy - which in our case concerns the right hand, and (2) the initiation of the pendulum release-which in our case concerns the left hand. Thus, the grip efficiency measured for a given subject on each and every trial reflected his/her true motor prediction for the forthcoming collision of that trial; and this was true whether the pendulum was released by the experimenter (task E) or by the subject (task S), since in both tasks the subjects were required to predict the magnitude of the collision between the pendulum and the hand-held load cell, in order to voluntarily and adequately increase the grip force level in time for the impact. Hence, in the theoretical framework of internal models, subjects used for the voluntary increase of grip force, the efference copy of the motor command from the right-hand action both in task $\mathrm{E}$ and in task S (cf. inner-right loop of Fig. 4). Our results revealed that in task E, the patients' responses-efficiency (safety margin) and accuracy of the predictive grip force adjustments (correlation and slopes of the individual regression lines)were similar to that observed in the controls. Moreover, and most importantly, our results showed that in task $\mathrm{S}$, for which the pendulum was self-released, motor prediction reached similar accuracy levels (correlation and slopes of the individual regression lines) in patients and in controls. Normal accuracy in predictive control of action has previously been shown in similar tasks (Delevoye-Turrell et al. 2002, 2003b; Carnahan et al. 1996; Saoud et al. 2000). This is an important point as it argues in favour of preserved and well functioning forward model motor predictions in schizophrenia. It also suggests that the efficiency deficits in task S for patients with schizophrenia cannot be simply explained by a general problem in motor prediction. Finally, the dissociation (accuracy vs. efficiency) revealed in our patients with schizophrenia demonstrates that forward model motor predictions cannot be the neural mechanisms that subserves the efficiency differences between self and externally initiated events in healthy controls.

Another relevant point to our discussion is the fact that the learning curves in the patients with schizophrenia follow similar learning functions than that observed in controls (Fig. 3). This is an important finding as it suggests that patients possess preserved low order comparator mechanisms for motor learning (cf. inner and outer-right loops of Fig. 4). In computing models, learning has in fact been defined as the dynamic evolution of the coupling between inverse and forward models that occurs through experience and which enables a more accurate and efficient planning of action in reference to the context (Kawato 1999). The data presented here all converge to suggest that the use of efferent and afferent information for the dynamic inverse/forward modelling of grip control is preserved in patients with schizophrenia. Thus, the efficiency difference between tasks $\mathrm{E}$ and $\mathrm{S}$ cannot be explained by the use of efference copy, as efference copy is available in both tasks, and is used in a similar fashion by both experimental groups to improve grip efficiency over time. This is why we suggest that it is important to consider the difference in the efficiency levels used between tasks $\mathrm{E}$ and $\mathrm{S}$ (and not simply the absolute level of efficiency) to point out the very effect of being-or not-the initiator of an action. We further propose that the efficiency difference between self initiated and externally initiated events can be a relevant and important indicator of the functional status of the pre-reflective sense of agency.

Both controls and patients were the most efficient when provided with qualitative feedback. Hence, feedback seems to have provided subjects with a better awareness of the 


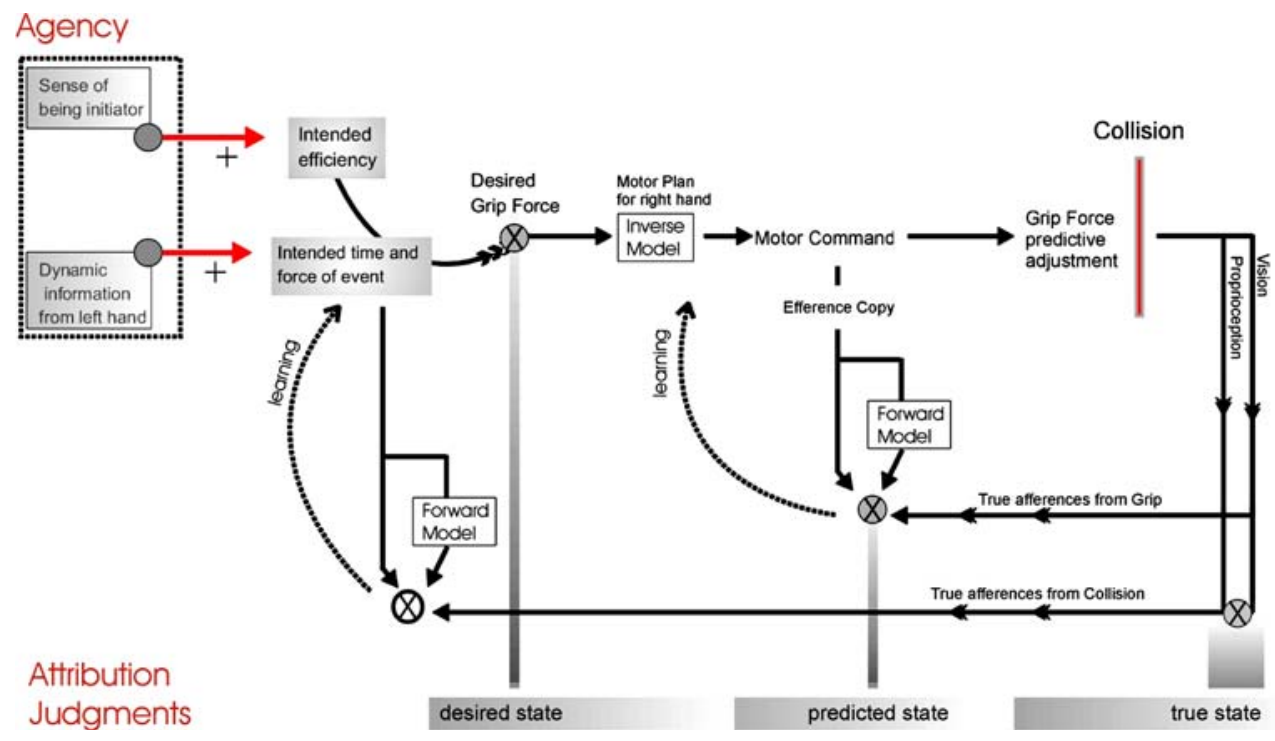

Fig. 4 Illustrates the forward model predictive mechanisms that provides an optimal control of grip force efficiency. The desired grip force level $(G F)$ is determined by combining an "Intended efficiency" with an "Intended time and force of collision". This desired GF state is sent to a Feedforward controller (inverse model) that generates a set of motor commands. This is then transformed into a set of muscle commands that reaches the peripheral effectors (e.g. muscles of the arm and fingers) after a given efference delay, and before the critical event (i.e. collision). The Dynamic predictor (forward model), which receives a copy of the motor commands (efference copy), is the centre that generates the predicted sensory feedback or internal feedback. To ensure that the executed movement is equal to the desired one, the actual sensory feedback (obtained here through vision and proprioception) is compared to the internal feedback in order to compute an error signal or

efficiency levels that were used. Nevertheless, this improvement in motor awareness had no effect on the indicator of the sense of agency. Indeed, the efficiency difference between tasks $\mathrm{E}$ and $\mathrm{S}$ was similar with or without feedback. This was true for both controls and patients. These results suggest that the pre-reflective sense of agency is not sensitive to voluntary control and argue in favour of an automatic (non-intentional) nature to its process. On the other hand, when considering the closeness of fit between subjective and objective report of GF efficiency, we observed that controls had greater correlation values in task $\mathrm{S}$ than in task $\mathrm{E}$ whereas it was similar for patients. Thus, it is possible that the low order sense of agency may have an influence on the more integrated processes that give rise to motor awareness. Because of a deficit of the pre-reflective agency, patients with schizophrenia would also be impaired in their awareness of the levels of GF efficiency set at the time of impact. Overall, these results suggest that the sense of agency might not follow a top-down process but could have a bottom-up effect on the consciousness of action. This is an interesting hypothesis as it would suggest that the deficits in motor and perceptual awareness as well as the problems in self-recognition tasks in schizophrenia may all sensory discrepancy signal. If the sensory feedback from the produced movement is equal to that predicted, the two signals will cancel out. Conversely, if these signals are not equal, the sensory discrepancy will be used to render the pairs of inverse/forward models more accurate and thus, improve the prediction of future movements. This process is symbolised here by the learning arrows, for the accuracy of GF control (outer loop), and for the efficiency of GF control (inner loop). For selfinitiated collisions, the accuracy of the "Intended time and force of event" is improved with the presence of dynamic information from the left hand (release of the pendulum) for both the controls and the patients. The level of "Intended efficiency" may be improved by a fixed amount by the presence of a "Agency signal", which was shown to be present in healthy controls only

be caused by a deficit of a low order mechanisms subserving a sense of agency at a pre-reflective level.

But what may be the mechanisms by which healthy controls are able to more efficiently adjust their grip when they release the pendulum themselves? From a pure motor control standpoint, when healthy controls release the pendulum with their left hand, brain structures receive critical information about the mass of the pendulum, the timing of the pendulum release, which globally provides the motor system with the necessary information to better synchronize the actions that are to-be-performed by both hands (illustrated by the "Dynamic information from the left hand", left of Fig. 4). However, we suggest that when one self-releases the pendulum, one can in addition improve the efficiency control of the action because one experiences the pendulum release from a first-person perspective, i.e. "I have the feeling of being in control of the initiation of this event". This feeling of being in control can help amongst other things the confidence level that subjects have about, e.g. the dynamical properties of the self-initiated collision. This would in turn encourage subjects to decrease the excessive grip force used, i.e. reduce by a fixed-amount the safety margin very early during action planning, in order to improve adequacy between own actions 
and self-initiated events (illustrated by the "Sense of being initiator", left side of Fig. 4). Even if this intriguing topic requires a lot more research, the results reported here suggest that (1) the motor control of pendulum release is necessary for better timing and more precise scaling of grip force to predicted load force modulations, but that (2) the sense of being the agent is required to decrease the pre-set safety margin in such a way to render grip force more efficiently adjusted in self-initiated compared to externally initiated events. Our results further suggest that schizophrenia is a pathological illness that is not concerned by a problem of motor integration for the predictive control of grip but is rather associated with an impaired sense of agency at the immediate and pre-reflective level of experience.

Our results fit well with Tsakiris and Haggard's data (2003) and also set in well within the more cognitive theories of schizophrenia (e.g. van Hoof 2004). More specifically, a nice parallel can be made between our results and the two types of sensory attenuation-proportional and fixed-proposed by Tsakiris and Haggard (2005). The proportional sensory attenuation corresponds in our case to the efficiency difference that was observed as a consequence of improved forward model prediction by: (1) experience through time and (2) the presence of feedback. These two means helped the motor system to decrease the degree of discrepancy between the predicted and the true afferent information from action generation. Here, this proportional sensory attenuation, which is based on motor prediction and on the comparison of predicted and true status, is preserved in schizophrenia. The fixed sensory attenuation on the other hand would correspond to the fact of being the initiator of the release and set the general contextual modulation to "I am the agent of pendulum release". In absence of this input contextual parameter (see Fig. 4 left), GF scaling would be planned as if the pendulum were released by an external agent. From this, one must consider the possibility that a pre-reflective sense of agency is subserved by an efferent-driven process that is different from those involved in the predictive control of movement, even if they must be tightly linked for common function.

Alternative interpretations may be considered for the results presented here. First, our results could have been the consequence of motor dyscoordination. However, coordination scores (gross motor coordination-see Table 2) were not correlated to the efficiency difference between task $\mathrm{E}$ and $\mathrm{S}$. In addition, the delay between time of impact and time of maximum grip force (fine motor coordination) was similar for patients and controls. Above all, if this hypothesis were true, patients should have been less efficient in task $\mathrm{S}$ because it required more coordination than task E. It is also important to note that the similar time delay between impact and peak grip force in both experimental groups suggest that the patients' results can not be explained by an impaired prediction of the time of collision. Second, a deficit in the allocation of attention could have explained the poor efficiency level reached in task $\mathrm{S}$, as it required more attention than task E-indeed, for task S, subjects were required to allocate attention to the initial position of the pendulum, to the auditory signal and to the need of optimising grip efficiency; for task $\mathrm{E}$, subjects allocated attention only to the auditory signal and to grip efficiency. However, the indicator for the sense of agency was not correlated to the patients' scores in the attentional capacity tests (Table 2). Moreover, if this attentional hypothesis were true, schizophrenic patients should have been most efficient in task E. This was not the case. Finally, patients' results could have been heterogeneous. Indeed, some patients could have performed as controls i.e. more efficient in $\mathrm{S}$ than in E, whereas the others on the contrary could have been more efficient in $\mathrm{E}$ than in S. However, for both subgroups presented in Table 2 (high vs. low performers in the various clinical tests), there was a similar absence of efficiency difference between task $\mathrm{E}$ and task S.

Our study is original in the fact that it does not explore judgements of attribution, but points specifically to the prereflective and efferent-driven sense of agency in patients with schizophrenia. Hence, at the very basic level of action awareness, we have shown that patients with schizophrenia are impaired in their first-order experience of being the author of self-initiated actions. To our knowledge, this is the first study to argue in favour of an impaired pre-reflective sense of agency in paranoid schizophrenia. Moreover, we bring strong experimental evidence for the theoretical hypothesis presented by Tsakiris and Haggard (2005), which suggests that the efferent-driven sense of agency, even if related to, must be distinct from forward motor prediction. This pre-reflective sense of agency does not seem to be accessible to voluntary top-down control, but it would play an important role in facilitating access to the more conscious levels of motor awareness.

We acknowledge the possibility that the processes underlying the pre-reflective sense of agency are fundamentally different from the more global processes involved in self-recognition tasks (Tsakiris et al. 2005). For example, judgements of attribution require high-level integrative mechanisms in order to group elements of various nature and from multiple sources (e.g. visual, auditory, proprioceptive afferences with internal representations maintained in memory). In fact, functional integrative processes are required for the comparison that takes place during afferent-efferent matching, a process that plays an important role in attribution tasks. On the other hand, we have shown that the pre-reflective sense of agency is a low-order process that occurs at an early stage of action planning and that it is purely efferent-driven. Nevertheless, agency and selfrecognition must be tightly related. Experimental studies 
have shown that for healthy controls agency can improve performance-levels in self-recognition tasks, especially in the case where visual information is experimentally biased (Tsakiris et al. 2005). Thus, the pre-reflective agency could be an input that would provide the means to decide whether "the action I see is mine or not". This has been studied and extensively discussed within theoretical models of reflective sense of agency, e.g. Wegner's work (Aart et al. 2005; Wegner 2005, 2003; Wegner et al. 2004). In patients with schizophrenia, it has been reported that performances in self-recognition tasks are normal in situations where nonbiased visual feedback is provided. However, their performance level drops abnormally in the presence of biased visual information (Daprati et al. 1997). What could have provoked in these patients the drop in performance? Could it be because at a low-level of the nervous system, the dysfunctional sense of agency did not provide the patients with the means to cope with unreliable information from the environment? Here, we have reported data suggesting that at an implicit level, patients pre-reflectively experience self-initiated events as similar to externally imposed events. However, at the explicit level i.e. when asked, patients did not make attribution errors. This is in agreement with the above-mentioned studies: in absence of biased information from the environment (e.g. visual information), patients with schizophrenia are not impaired in judgement tasks.

An operational hypothesis is that the pre-reflective sense of agency is one of the many components of judgements of attribution. In schizophrenia, performance levels in judgement tasks need the integration of information from multiple sources, and may be preserved as long as afferent information (e.g. visual and auditory contextual information) provide the means to compensate the dysfunctional prereflective and efferent-driven sense of agency. Hence, with biased visual feedback, laboratory set experiments using judgement tasks may have revealed punctually, and at an integrated reflective level, a permanent and low order deficit of the pre-reflective sense of agency in schizophrenia. Interestingly, the fluctuations of passivity phenomenon in schizophrenia could be explained by the punctual revelation of a permanent deficit of the pre-reflective sense of agency when compensatory mechanisms are not available. By gaining a better understanding of what exactly triggers passivity phenomena under real life situations, it could be possible to reduce the occurrence of passivity phenomena in patients with schizophrenia by emphasising during rehabilitation programs the importance of compensatory mechanisms.

\section{Conclusion}

We report data, which reveal that patients with schizophrenia adjust anticipatory grip force responses as accurately in time and force than control subjects both in self-initiated and in externally initiated collisions. However, contrary to controls, they do not improve grip force efficiency when they are agent of pendulum release. This finding strongly argues in favour of a deficit of the pre-reflective sense of agency in patients with paranoid schizophrenia. This prereflective sense may rely on a low order process, which would intervene at a very early stage of action planning but which would be distinct from motor prediction. We propose that clinical delusions of influence might be due to a punctual revelation of this permanent deficit, when sensory compensatory mechanisms are not available. Further studies are needed to better specify the functional relation that must relate the deficits reported here in the pre-reflective sense of agency with those previously reported in judgments of attribution tasks for patients with paranoid schizophrenia.

Acknowledgments Acknowledgments are given to the French Medical Research Foundation for their financial help. Many thanks to S. Gallagher, to A. Giersch and to three anonymous reviewers for their useful comments.

\section{References}

Aart H, Custers R, Wegner DM (2005) On the inference of personal authorship: enhancing experienced agency by priming effect information. Consciousness and Cognition 14:439-458

Blakemore S-J, Wolpert D, Frith C (1998) Central cancellation of selfproduced tickle sensation. Nat Neurosci 7:635-640

Blakemore S-J, Smith J, Steel R, Johnstone CE, Frith CD (2000) The perception of self-produced sensory stimuli in patients with auditory hallucinations and passivity experiences: evidence for a breakdown in self-monitoring. Psychol Med 30:1131-1139

Blakemore S-J, Wolpert D, Frith C (2002) Abnormalities in the awareness of action. Trends Cogn Sci 6:237-242

Carnahan H, Elliott D, Velamoor VR (1996) Influence of object size on prehension in leukotomized and unleukotomized individuals with schizophrenia. J Clin Exp Neuropsychol 8:136-147

Daprati E, Franck N, Georgieff N, Proust J, Pacherie E, Dalery J, Jeannerod M (1997) Looking for the agent: an investigation into consciousness of action and self consciousness in schizophrenic patients. Cognition 61(1):71-86

Delevoye-Turrell YN, Giersch A, Danion JM (2002) A deficit in the adjustment of grip force responses in schizophrenia. NeuroReport 13(12):1537-1539

Delevoye-Turrell YN, Li FX, Wing AM (2003a) Efficiency of grip force adjustment for impulsive loading. Quart J Exp Psychol 56A(7):1113-1128

Delevoye-Turrell YN, Giersch A, Danion JM (2003b) Abnormal sequencing of motor action in patients with schizophrenia: evidence from grip force adjustments during slow and impulsive loading conditions. Am J Psychiatry 160:134-141

Feinberg I (1978) Efference copy and corollary discharge: implications for thinking and its disorders. Schizophr Bull 4(4):636-640

Franck N, Farrer C, Georgieff N, Marie-Cardine M, Dalery J, d'Amato T, Jeannerod M (2001) Defective recognition of one's own actions in patients with schizophrenia. Am J Psychiatry 158(3):454-459

Frith CD (1992) The cognitive neuropsychology of schizophrenia. Lawrence Erlbaum, Mahwah 
Frith CD, Blakemore S-J, Wolpert DM (2000) Explaining the symptoms of schizophrenia: abnormalities in the awareness of action. Brain Res Brain Res Rev 31(2-3):357-363

Gallargher S (2000) Philosophical conceptions of the self: the implication for cognitive science. Trends Cogn Sci 4(1):14-21

Gallagher S (2004) Neurocognitive models of schizophrenia: a neurophenomenological critique. Psychopathology 37(1):8-19

Kawato M (1999) Internal models for motor control and trajectory planning. Curr Opin Neurobiol 9:718-727

Saoud M, Coello Y, Dumas P, Franck N, D'Amato T, Dalery J, Rossetti Y (2000) Visual pointing and speed/accuracy trade-off in schizophrenia. Cogn Neuropsychiatry 5:123-134

Shergill S, Bays P, Frith CD, Wolpert DM (2003) Two eyes for an eye: the neuroscience of force escalation. Science 301(5630): 187

Shergill SS, Samson G, Bays PM, Frith CD, Wolpert DM (2005) Evidence for sensoryprediction deficits in schizophrenia. Am J Psychiatry 162(12):2384-2386

Shoemaker S (1984) Identity, cause, and mind. Cambridge University Press, Cambridge

Sperry RW (1950) Neural basis of spontaneous optokinetic response produced by visual inversion. J Comp Physiol Psychol 43:482-489

Tsakiris M, Haggard P (2003) Awareness of somatic events following a voluntary action. Exp Brain Res 149:439-446

Tsakiris M, Haggard P (2005) Experimenting with the acting self. Cogn Neuropsychol 22(3/4):387-407

Tsakiris M, Haggard P, Franck N, Mainy N, Sirigu A (2005) A specific role for efferent information in self-recognition. Cognition 96(3):215-231
Turrell YN (2000) Grip force adjustments in collisions. PhD Thesis; Birmingham University Press, pp 46-48

Turrell YN, Li FX, Wing AM (2001) Estimating the minimum grip force required when grasping objects under impulsive loading conditions. Behav Res Methods Instrum Comput 33(1):38-45

Van Hoof JJM (2004) The abnormal development of drive and guidance mechanisms in the brain: the pathogenesis of schizophrenia. Acta Neuropsychiatrica 14(3):134-146

Von Holst E, Mittelstaedt H (1950) Das reaffernzprinzip wechselwirkungen zwichen zentainervensystem und peripherie. Naturwissenschalten 37:464-476

Westling G, Johansson RS (1984) Responses in glabrous skin mechanoreceptors during precision grip in humans. Exp Brain Res 66:128-140

Wegner DM (2003) The mind's best trick: how we experience conscious will. Trends Cogn Sci 7:65-69

Wegner DM (2005) Who is the controller of controlled processes. In: Hassin R, Uleman JS, Bargh JA (eds) The new unconscious. Oxford University Press, New York, pp 19-36

Wegner DM, Sparrow B (2004) Authorship processing. In: Gazzaniga $M$ (ed) The new cognitive neurosciences. MIT Press, Cambridge

Wegner DM, Sparrow B, Winerman L (2004) Vicarious agency: Experiencing control over the movements of others. J Pers Soc Psychol 86:838-848 